\title{
Adaptive Local Polynomial Fourier Transform in ISAR
}

\author{
Igor Djurović, ${ }^{1}$ Thayananthan Thayaparan, ${ }^{2}$ and Ljubiša Stankovićc \\ ${ }^{1}$ Electrical Engineering Department, University of Montenegro, 81000 Podgorica, Serbia and Montenegro \\ ${ }^{2}$ Department of National Defence, Defence R \& D Canada - Ottawa, 3701 Carling Avenue, \\ Ottawa, ON, Canada K1A 0Z4
}

Received 23 May 2005; Revised 14 November 2005; Accepted 15 November 2005

\begin{abstract}
The adaptive local polynomial Fourier transform is employed for improvement of the ISAR images in complex reflector geometry cases, as well as in cases of fast maneuvering targets. It has been shown that this simple technique can produce significantly improved results with a relatively modest calculation burden. Two forms of the adaptive LPFT are proposed. Adaptive parameter in the first form is calculated for each radar chirp. Additional refinement is performed by using information from the adjacent chirps. The second technique is based on determination of the adaptive parameter for different parts of the radar image. Numerical analysis demonstrates accuracy of the proposed techniques.
\end{abstract}

Copyright ( 2006 Hindawi Publishing Corporation. All rights reserved.

\section{INTRODUCTION}

The inverse synthetic aperture radar (ISAR) has attracted wide interest within scientific and military community. Some ISAR applications are already well known and studied. However, many important issues remain to be addressed. For example, suitable enhancement technique for the fast maneuvering radar targets or targets with fast moving parts is not yet known. Also, standard approaches based on the Fourier transform (FT) fail to resolve influence of close reflectors. There are several techniques for improvement of the ISAR radar image in the case of fast maneuvering targets or in the case of objects with complex reflector geometry. Here we mention only two groups of such enhancement techniques as follows:

(i) techniques that adopt transform parameters for assumed parametric target motion model [1],

(ii) techniques where reflection signal components are parametrized, while the signal components caused by reflectors are estimated by using some of well developed parametric spectral estimation tools $[2,3]$.

Both of these techniques have some advantages, but also some drawbacks for specific applications. The first group of techniques is strongly based on radar target geometry with assumed motion model. These techniques could become inaccurate in the case of a changing motion model. The second group of techniques is tested on simulated examples.
However, its application in real scenarios, where signal components are caused by numerous scatterers, could be very difficult. Namely, there are no appropriate methods for parameters estimation of signals with a very large number of components.

In this paper we propose a modification of the first group of research techniques. The adaptive local polynomial Fourier transform (LPFT) is used. Adaptive coefficients are calculated for each considered chirp in the radar signal mixture. It is important to note that the proposed technique does not assume any particular model of radar target motion. The adaptive parameters are estimated for each scattering point independently. Based on the analysis of the signal obtained from the target we consider some simplifications in the process of calculation of the adaptive transform. In this way we keep the calculation burden within reasonable limits. Two techniques for enhancement of the radar image by using the LPFT are considered. The first one is based on information obtained from each chirp separately and on possible refinement by combining results from various chirps. The second technique is based on detection of regions of interest in the range/cross-range plane and on determination of the optimal LPFT for each detected region.

The paper is organized as follows. The target and radar signal modeling is discussed in Section 2. The proposed methods are introduced in Section 3. Simulation study is given in Section 4. 


\section{RADAR SIGNAL MODEL}

Consider a radar signal consisting of $M$ continuous wave coherent pulses:

$$
v_{M}(t)=\sum_{m=0}^{M-1} v_{0}\left(t-m T_{r}\right),
$$

where $v_{0}(t)$ is basic impulse limited within the interval $-T_{r} / 2 \leq t<T_{r} / 2$. The linear frequency modulated (FM) signal is used in our simulations as a basic impulse: $v_{0}(t)=$ $\exp \left(j \pi B t^{2} / T_{r}\right)$, where $B$ is bandwidth control parameter while $T_{r}$ is pulse repetition time. Alternative radar model used in practice has radar pulses with stepped frequencies. Defocusing effect considered in this paper and timefrequency $(\mathrm{TF})$ signatures of obtained radar signals have similar behavior for these two forms of radar signals $[4,5]$.

Signal emitted toward radar target can be written as

$$
u(t)=e^{j 2 \pi f_{0} t} v_{M}(t)
$$

where $f_{0}$ is radar operating frequency. Received signal, reflected from single reflector target at distance $d(t)$, is delayed for $2 d(t) / c$, with $c$ being propagation rate:

$$
u_{R}(t)=\sigma u\left(t-\frac{2 d(t)}{c}\right)
$$

Demodulation of received signal can be performed by multiplying received with transmitted signal $u(t)$ :

$$
\begin{aligned}
q(t)= & \sigma u^{*}\left(t-\frac{2 d(t)}{c}\right) u(t) \\
= & \sigma \exp \left(\frac{j 4 \pi}{c} f_{0} d(t)\right) \sum_{m=0}^{M-1} v_{0}^{*}\left(t-\frac{2 d(t)}{c}-m T_{r}\right) \\
& \times \sum_{m=0}^{M-1} v_{0}\left(t-m T_{r}-T_{0}\right) .
\end{aligned}
$$

Parameter $T_{0}$ is used in radar imaging for compensation of target distance. For properly selected $T_{0}$ and after highpass filtering, the signal $q(t)$ can be approximately written as

$$
\begin{aligned}
q(t) \approx & \sigma \exp \left(\frac{j 4 \pi}{c} f_{0} d(t)\right) \\
& \times \sum_{m=0}^{M-1} v_{0}^{*}\left(t-\frac{2 d(t)}{c}-m T_{r}\right) v_{0}\left(t-m T_{r}\right) \\
= & \sum_{m=0}^{M-1} q(m, t)
\end{aligned}
$$

where

$$
\begin{aligned}
q(m, t) & =\sigma \exp \left(\frac{j 4 \pi}{c} f_{0} d(t)\right) v_{0}^{*}\left(t-\frac{2 d(t)}{c}-m T_{r}\right) v_{0}\left(t-m T_{r}\right), \quad t \in\left[\left(m-\frac{1}{2}\right) T_{r},\left(m+\frac{1}{2}\right) T_{r}\right) \\
& =\sigma \exp \left(\frac{j 4 \pi}{c} f_{0} d(t)\right) \exp \left(\frac{j 4 \pi B}{c T_{r}} d(t)\left(t-m T_{r}\right)\right) \exp \left(-\frac{j \pi B}{T_{r}}\left(\frac{2 d(t)}{c}\right)^{2}\right)
\end{aligned}
$$

Keeping in mind $B \ll f_{0}$, we can neglect $\exp (-j \pi B(2 d(t) /$ $c)^{2} / T_{r}$ ) with respect to other two components. The value of $q(m, t)$ can approximately be written as

$$
\begin{aligned}
& q(m, t) \\
& \quad \approx \sigma \exp \left(\frac{j 4 \pi}{c} f_{0} d(t)\right) \exp \left(\frac{j 4 \pi B}{c T_{r}} d(t)\left(t-m T_{r}\right)\right) .
\end{aligned}
$$

This signal is commonly given in the form

$$
\begin{aligned}
q(m, \tau) \approx & \sigma \exp \left(\frac{j 4 \pi}{c} f_{0} d\left(\tau+m T_{r}\right)\right) \\
& \times \exp \left(\frac{j 4 \pi B d}{c T_{r}}\left(\tau+m T_{r}\right) \tau\right)
\end{aligned}
$$

where $t=\tau+m T_{r}$. Parameter $\tau \in\left[-T_{r} / 2, T_{r} / 2\right)$ is referred to as fast-time, while $m=0,1, \ldots, M-1$, is called slow-time coordinate. Commonly, in actual radar systems, signals are discretized in fast-time coordinate with sampling rate $T_{s}=T_{r} / N, \tau=n T_{s}$, where $n \in[-N / 2, N / 2)$. However, due to notational simplicity we will keep continuous fasttime coordinate. Classical radar setup assumes that the radar target position is a linear function of time $d(t)=D_{0}+V t$. Then the radar model produces

$$
\begin{aligned}
q(m, \tau) \approx & \sigma \exp \left(\frac{j 4 \pi}{c} f_{0}\left[D_{0}+V\left(\tau+m T_{r}\right)\right]\right) \\
& \times \exp \left(\frac{j 4 \pi B}{c T_{r}}\left[d_{0}+V\left(\tau+m T_{r}\right)\right] \tau\right) \\
= & \sigma \exp \left(\frac{j 4 \pi}{c} f_{0}\left(D_{0}+V \tau\right)\right) \\
& \times \exp \left(\frac{j 4 \pi V m}{c}\left(f_{0} T_{r}+B \tau\right)\right) \\
& \times \exp \left(\frac{j 4 \pi \tau B}{c T_{r}}\left(D_{0}+V \tau\right)\right) .
\end{aligned}
$$




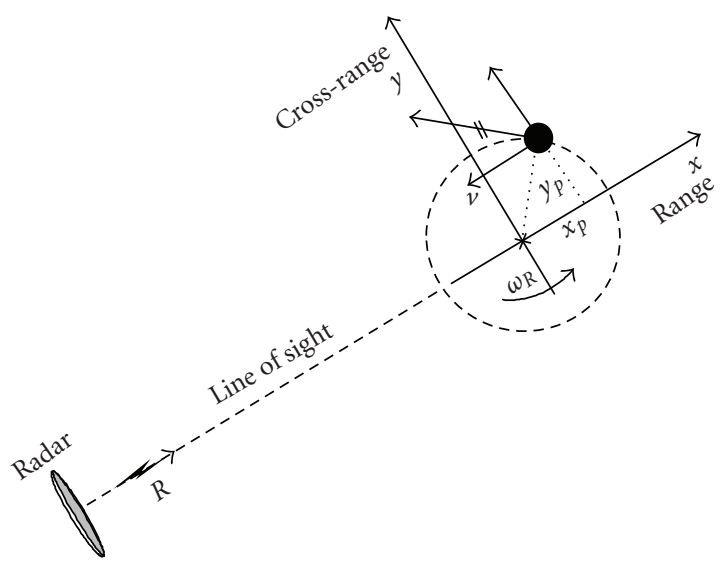

FIGURE 1: Illustration of the radar target geometry.

Since $f_{0} \gg B, T_{r}>|\tau|$, and $D_{0} \gg V \tau$, signal $q(m, \tau)$ can be further simplified to

$$
\begin{aligned}
q(m, \tau) \approx & \sigma \exp \left(\frac{j 4 \pi f_{0} D_{0}}{c}\right) \exp \left(\frac{j 4 \pi V m f_{0} T_{r}}{c}\right) \\
& \times \exp \left(\frac{j 4 \pi \tau B D_{0}}{c T_{r}}\right) .
\end{aligned}
$$

A two-dimensional (2D) FT of this signal over $m$ and $\tau$ is approximately

$$
\begin{aligned}
& Q\left(\omega_{\tau}, \omega_{m}\right) \\
& =\int_{\tau} \sum_{m=0}^{M-1} q(m, \tau) e^{-j \omega_{\tau} \tau-j \omega_{m} m} d \tau \\
& \approx(2 \pi) \sigma \exp \left(\frac{j 4 \pi f_{0} D_{0}}{c}\right) \delta\left(\omega_{\tau}-\frac{4 \pi B D_{0}}{c T_{r}}\right) \\
& \quad \times \frac{\sin \left(\left(\omega_{m}-4 \pi V f_{0} T_{r} / c\right) M / 2\right)}{\sin \left(\left(\omega_{m}-4 \pi V f_{0} T_{r} / c\right) / 2\right)} e^{-j\left(\omega_{m}-4 \pi V f_{0} T_{r} / c\right)(M-1) / 2} .
\end{aligned}
$$

For large $M$ we can write the magnitude of $Q\left(\omega_{\tau}, \omega_{m}\right)$ as

$$
\begin{aligned}
& \left|Q\left(\omega_{\tau}, \omega_{m}\right)\right| \\
& \quad \approx(2 \pi) \sigma \delta\left(\omega_{\tau}-\frac{4 \pi B D_{0}}{c T_{r}}\right) M \delta\left(\omega_{m}-\frac{2 V f_{0} T_{r}}{c}\right) .
\end{aligned}
$$

For rotating scatterer given in Figure 1, distance can approximately be written as $d(t) \approx R(t)+x_{p} \cos (\theta(t))+y_{p} \sin (\theta(t))$, where $R(t)$ is distance of the target rotation center from the radar, where coordinates of the scatterer, for $\tau=0$, are $\left(x_{p}, y_{p}\right)$. Coordinate system is formed in such a way that the coordinate $x$ is the line of sight. Assume constant rotation velocity $\theta(t)=\omega_{R} t$, with relatively small angular movement of the target $\left|\omega_{R} T_{r}\right| \ll 1$ (it implies that $\cos (\theta(t)) \approx 1$ and $\sin (\theta(t)) \approx 0)$. According to the introduced conditions, $d(t) \approx x_{p}$ and $v(t)=d^{\prime}(t)=-x_{p} \theta^{\prime}(t) \sin (\theta(t))+$ $y_{p} \theta^{\prime}(t) \cos (\theta(t)) \approx y_{p} \theta^{\prime}(t) \cos (\theta(t)) \approx y_{p} \omega_{R}$. Commonly, it is assumed that $R(t)$ is compensated by adjusting $T_{0}$ in (4). Thus, we will not consider it in our algorithm. Then $\left|Q\left(\omega_{\tau}, \omega_{m}\right)\right|$ can be written as

$$
\begin{aligned}
& \left|Q\left(\omega_{\tau}, \omega_{m}\right)\right| \\
& \quad \approx(2 \pi) \sigma M \delta\left(\omega_{\tau}-\frac{4 \pi B x_{p}}{c T_{r}}\right) \delta\left(\omega_{m}-\frac{4 \pi y_{p} \omega_{R} f_{0} T_{r}}{c}\right) \\
& \quad=(2 \pi) \sigma M \delta\left(\omega_{\tau}-c_{1} x_{p}\right) \delta\left(\omega_{m}-c_{2} y_{p}\right) .
\end{aligned}
$$

It represents the ISAR image of scatterer $\left(x_{p}, y_{p}\right)$ for a given instant under introduced assumptions. Note that the constants that determine resolution of the radar image are given by $c_{1}=4 \pi B /\left(c T_{r}\right)$ and $c_{2}=4 \pi \omega_{R} f_{0} T_{r} / c$. The radar image is formed as superposition of radar images of all scatterers $\left(x_{p}, y_{p}\right), p=1,2, \ldots, P$. It is approximately given as

$$
\left|Q\left(\omega_{\tau}, \omega_{m}\right)\right|=\sum_{p=1}^{P}(2 \pi) \sigma_{p} \delta\left(\omega_{\tau}-c_{1} x_{p}\right) \delta\left(\omega_{m}-c_{2} y_{p}\right),
$$

where $\sigma_{p}$ is the reflection coefficient that corresponds to the $p$ th scatterer point.

In numerous cases we cannot assume that the radar model can be simplified in the previously described manner. For example, radar target can be very fast, or model of radar target motion can be more complicated (e.g., 3D motion). Then, instead of complex sinusoids given by (10) we will get that components corresponding to particular scatterers are 
polynomial phase signals:

$$
q(m, \tau)=\sigma_{p} \exp \left(j \sum_{l=0}^{L} \frac{a_{m, l} \tau^{l}}{l !}\right),
$$

where parameters $a_{m, l}$ depend on the considered chirp and scatterer motion. For example, for the target motion model $d(t)=D_{0}+V_{0} t+A t^{2} / 2$, where $A$ is acceleration of target, coefficients $a_{m, l}$ are approximately equal to

$$
\begin{aligned}
& a_{m, 0}=\frac{4 \pi}{c} f_{0}\left(D_{0}+m T_{r}+\frac{m^{2} T_{r}^{2}}{2}\right), \\
& a_{m, 1}=\frac{4 \pi}{c}\left(f_{0} V_{0}+f_{0} A m T_{r}+\frac{B D_{0}}{T_{r}}+B V_{0} m+\frac{B A m^{2} T_{r}}{2}\right), \\
& a_{m, 2}=\frac{8 \pi}{c}\left(f_{0} \frac{A}{2}+B \frac{V_{0}}{T_{r}}+A m\right), \\
& a_{m, 3}=\frac{12 \pi B A}{c T_{r}},
\end{aligned}
$$

and $a_{m, l}=0$ for $l>3$. Some terms of these coefficients can be neglected, but in general it is not simple as in the case when we can assume that the scatterer position is a linear function. Situation becomes even more difficult in the case when target model is not a simple rotating model. Then, very complicated relationship between position of scatterers $\left(x_{p}, y_{p}\right)$ and coefficients of the polynomial in the signal phase can be established. Also, polynomial that should be used to accurately estimate signal phase is of very high order. Radar image obtained by using the 2D FT of signal with higher order polynomial becomes spread (defocused) in the range/crossrange domain $\left(\omega_{\tau}, \omega_{m}\right)$. The goal of ISAR signals processing is to obtain a focused radar image, that is, to remove influence of the higher order polynomial in signal phase of each component.

Usually, it is assumed that modeling of coefficients is possible based on the target motion model. In that case, instead of all possible parameters, only parameters of the motion model should be used in order to perform enhancement of the radar image.

The first group of techniques for enhancement of radar images is based on this concept. One such approach is described in [6] where it is assumed that radar scatter can be modeled with relative simple motion model which assumes that velocity increases or decreases linearly (or that angular velocity changes in linear manner) within repetition time. After estimating acceleration of target, variation in the velocity is compensated from signal and finally focused radar image is obtained. It corresponds to removing influence of acceleration from (15). However, these techniques are very sensitive to any variations from assumed motion model. They cannot be used for 3D motion models.

Alternative techniques are based on estimation of all coefficients in the polynomial of all components in the received signal $[2,3]$. These techniques are usually based on iterative removing of the lower order coefficients from signal phase in order to estimate the highest order coefficient. Then, estimation of lower order coefficients is performed by using the same procedure but for dechirped signal. It means that error in estimation of the highest order coefficient propagates toward lower order coefficients. Furthermore, it has recently been shown that these procedures are biased for multicomponent signals and that dechirping procedure used to produce signal suitable for estimation of lower order coefficients introduces additional source of errors for multicomponent signals. These techniques are also time consuming and, as far as we know, never applied to signals with large number of components. Numerous components caused by target scatterers could appear in radar signal.

A novel technique for enhancement of radar images, that introduces just one new adaptive parameter in the FT expression for each received signal, is introduced in the next section. For each chirp only one parameter of the transform should be estimated. The second important property of this technique is in the fact that we do not assume any particular motion model. It can be applied for any realistic motion of targets.

\section{ADAPTIVE LOCAL POLYNOMIAL FT}

In this section we introduce the LPFT as a tool for the ISAR image autofocusing. Two forms of the adaptive LPFT are proposed. The first form can be applied to each chirp component separately with possible refinement by using information from the adjacent chirps (Section 3.1). The second form performs evaluation of the adaptive LPFT for each detected region of interest in the radar image (Section 3.2).

\subsection{First form: adaptive LPFT for radar signals}

In order to develop this approach we will go through several typical cases of signals, starting from a very simple and going toward more complicated ones. Improvement in signal components concentration (focusing radar image) is performed by estimation of signal parameters without assuming any particular motion model. This is quite different approach comparing to the methods with predefined motion model or to the methods where estimation is performed for each parameter $a_{m, l}$.

\subsubsection{Linear FM signal case}

The simplest case of monocomponent linear FM signal

$$
q(m, \tau)=\sigma \exp \left(j\left[a_{m, 0}+a_{m, 1} \tau+\frac{a_{m, 2} \tau^{2}}{2}\right]\right)
$$

is considered first. In this case, dependence on $m$ in parameter indices will be removed for the sake of notation brevity. Then, the signal can be written as

$$
q(m, \tau)=\sigma \exp \left(j\left[a_{0}+a_{1} \tau+\frac{a_{2} \tau^{2}}{2}\right]\right) .
$$


For analysis of this kind of signals we can use the LPFT $[7,8]$,

$$
\begin{aligned}
& F\left(\omega_{\tau}, m ; \alpha\right) \\
& \quad=\int_{-\infty}^{\infty} q(m, \tau) w(\tau) \exp \left(-\frac{j \alpha \tau^{2}}{2}\right) \exp \left(-j \omega_{\tau} \tau\right) d \tau,
\end{aligned}
$$

where $w(\tau)$ is a window function of the width $T_{w}, w(\tau)=0$ for $|\tau| \geq T_{w} / 2$.

The LPFT is ideally concentrated along the instantaneous frequency, for $\alpha=a_{2}$,

$$
\begin{aligned}
F\left(\omega_{\tau}, m ; a_{2}\right)= & \sigma \int_{-\infty}^{\infty} w(\tau) \exp \left(j\left[a_{0}+a_{1} \tau+\frac{a_{2} \tau^{2}}{2}\right]\right) \\
& \times \exp \left(-j \omega_{\tau} \tau-\frac{j a_{2} \tau^{2}}{2}\right) d \tau \\
= & \sigma e^{j a_{0}} \int_{-\infty}^{\infty} w(\tau) \exp \left(-j\left(\omega_{\tau}-a_{1}\right)\right) d \tau \\
= & \sigma e^{j a_{0}} W\left(\omega_{\tau}-a_{1}\right),
\end{aligned}
$$

where $W\left(\omega_{\tau}\right)=F T\{w(\tau)\}$. Function $F\left(\omega_{\tau}, m ; a_{2}\right)$ is highly concentrated around $\omega_{\tau}=a_{1}$, since the FT of common wide window functions (rectangular, Hamming, Hanning, Gauss) is highly concentrated around the origin (in our experiments window width is equal to the repetition rate $T_{w}=T_{r}$ ). Radar image can be obtained from $F\left(\omega_{\tau}, m ; a_{2}\right)$ for considered $a_{2}$ by evaluating $1 \mathrm{D}$ FT along the $m$-coordinate:

$$
Q\left(\omega_{\tau}, \omega_{m} ; a_{2}\right)=\sum_{m=0}^{M-1} F\left(\omega_{\tau}, m ; a_{2}\right) e^{-j \omega_{m} m}
$$

\subsubsection{Higher order polynomial FM signal}

For higher order polynomial signal,

$$
q(m, \tau)=\sigma \exp \left(j \phi_{m}(\tau)\right)=\sigma \exp (j \phi(\tau))
$$

the LPFT can be written as

$$
\begin{aligned}
& F\left(\omega_{\tau}, m ; \alpha\right) \\
& =\int_{-\infty}^{\infty} \sigma \exp (j \phi(\tau)) w(\tau) \exp \left(-\frac{j \alpha \tau^{2}}{2}\right) \\
& \quad \times \exp \left(-j \omega_{\tau} \tau\right) d \tau \\
& =\sigma \int_{-\infty}^{\infty} \exp \left(j \phi(0)+j \phi^{\prime}(0) \tau+\frac{j \phi^{\prime \prime}(0) \tau^{2}}{2}\right. \\
& \quad+\frac{j \phi^{\prime \prime \prime}(0) \tau^{3}}{3 !}+\cdots+\frac{j \phi^{(n)}(0) \tau^{n}}{n !} \\
& \left.\quad+\cdots-\frac{j \alpha \tau^{2}}{2}-j \omega_{\tau} \tau\right) w(\tau) d \tau .
\end{aligned}
$$

For $\phi^{(n)}(0)=0$ for $n>2$, we obtain highly concentrated LPFT for $\alpha=\phi^{\prime \prime}(0)$,

$$
F\left(\omega_{\tau}, m ; \phi^{\prime \prime}(0)\right)=\sigma \exp (j \phi(0)) W\left(\omega_{\tau}-\phi^{\prime}(0)\right) .
$$

The second derivative of the signal phase is commonly called chirp-rate parameter.

In the case when higher order derivatives are nonzero the LPFT will not be ideally concentrated and we will have some spread in the frequency domain caused by the FT of terms $\exp \left(j \phi^{\prime \prime \prime}(0) \tau^{3} / 3 !+\cdots+j \phi^{(n)}(0) \tau^{n} / n !+\cdots\right)$. The LPFT forms that can be used to remove effects of the higher order derivatives from signal phase are introduced in $[7,8]$. These techniques are computationally demanding and difficult for application in the ISAR imaging in the real time.

Alternative technique is proposed in [9]. It is the socalled order adaptive LPFT. The width of the signal's FT is used as indicator of the polynomial phase order. Namely, proper order and parameters of the LPFT are applied if its width in the frequency domain is close to the width of considered window function $W\left(\omega_{\tau}\right)$.

The algorithm for the order adaptive LPFT determination can be summarized as follows.

(i) It begins with the ordinary FT calculation (zero-order LPFT) in the first step. If the width of this transform in the frequency domain is equal to the window width, it means that the image is already focused and there is no need for the LPFT order increase. Otherwise, go to the next step.

(ii) Use the first-order LPFT form considered in this paper (19). If the width of the this transform in the frequency domain is equal to the window width, it means that the image is focused. If the LPFTs still have some spread we should introduce new parameter $\beta$ in the transform (next coefficient in the LPFT phase will be $-\beta \tau^{3} / 3$ !) and repeat operation.

This very simple idea could be used for signals with one or at most few components. In complex multicomponent signal cases, more sophisticated technique, based on the concentration measures, will be introduced in the next section.

\subsubsection{Concentration measure}

From derivations given above, it can be concluded that for a known chirp-rate parameter we can obtain a focused radar image (highly concentrated TF representation). Also, it can be seen that the ISAR imaging based on the LPFT for a known chirp-rate parameter is slightly more demanding than the standard ISAR imaging since in addition to the standard procedure it requires multiplication with the term $\exp \left(-j \alpha \tau^{2} / 2\right)$. The next question is how to determine a value of the parameter $\alpha$ which will produce highly concentrated images. There are several methods in open literature. Here, the concentration measures will be used [10-12]. Before we propose our concentration measure, some properties of the LPFT will be reviewed. The LPFT satisfies energy 
conservation property

$$
\begin{gathered}
\int_{-\infty}^{\infty}\left|F\left(\omega_{\tau}, m ; \alpha\right)\right|^{2} d \omega_{\tau} \\
=\int_{-\infty}^{\infty} F\left(\omega_{\tau}, m ; \alpha\right) F^{*}\left(\omega_{\tau}, m ; \alpha\right) d \omega_{\tau} \\
=\int_{-\infty}^{\infty} \int_{-\infty}^{\infty} \int_{-\infty}^{\infty} q\left(m, \tau_{a}\right) w\left(\tau_{a}\right) \\
\quad \times \exp \left(-\frac{j \alpha \tau_{a}^{2}}{2}\right) \exp \left(-j \omega_{\tau} \tau_{a}\right) \\
\quad \times q^{*}\left(m, \tau_{b}\right) w\left(\tau_{b}\right) \exp \left(\frac{j \alpha \tau_{b}^{2}}{2}\right) \\
\quad \times \exp \left(j \omega_{\tau} \tau_{b}\right) d \tau_{a} d \tau_{b} d \omega_{\tau} \\
=\int_{-\infty}^{\infty} \int_{-\infty}^{\infty} q\left(m, \tau_{a}\right) w\left(\tau_{a}\right) \exp \left(-\frac{j \alpha \tau_{a}^{2}}{2}\right) \\
\times \int_{-\infty}^{\infty}\left(m, \tau_{b}\right) w\left(\tau_{b}\right) \exp \left(\frac{j \alpha \tau_{b}^{2}}{2}\right) \\
\times \delta\left(\tau_{a}-\tau_{b}\right) d \tau_{a} d \tau_{b} \\
\int^{2}(\tau) d \tau .
\end{gathered}
$$

Consider now the measure $\int_{-\infty}^{\infty}\left|F\left(\omega_{\tau}, m ; \alpha\right)\right|^{\gamma} d \omega_{\tau}$ for $\gamma \rightarrow 0$. Assume that $F\left(\omega_{\tau}, m ; \alpha\right)$ is concentrated in a narrow region around the origin in the frequency domain,

$$
\left|F\left(\omega_{\tau}, m ; \alpha\right)\right|=0 \quad \text { for } \omega_{\tau} \geq \frac{\Omega}{2}
$$

Then, we obtain

$$
\lim _{\gamma \rightarrow 0} \int_{-\infty}^{\infty}\left|F\left(\omega_{\tau}, m ; \alpha\right)\right|^{\gamma} d \omega_{\tau}=\Omega .
$$

We can see that the considered measure is smaller in the case of signals concentrated in narrower intervals in the TF plane. Therefore, this type of measure can be used to indicate concentration of the TF representation. In a realistic scenario, where signal side lobes and noise exist within the entire interval, this measure with $\gamma=0$ cannot be used, since it will produce approximately constant value. In order to handle this issue, we can use $0<\gamma<2$ instead of $\gamma=0$. As a good empirical value in our analysis we adopted $\gamma=1$. Accurate results can be achieved for a wider region of $\gamma \in[0.5,1.5]$.

The concentration measure based on the above analysis can be written as

$$
H(m, \alpha ; \gamma)=\frac{1}{\int_{-\infty}^{\infty}\left|F\left(\omega_{\tau}, m ; \alpha\right)\right|^{\gamma} d \omega_{\tau}} .
$$

Highly concentrated signal will be represented by a higher value of concentration measure (28). This concentration measure has been proposed [11] where it is analyzed in detail and compared with other concentration measures. This concentration measure produces accurate results for multicomponent signals, as well.

\subsubsection{Estimation of the chirp rate based on the concentration measure}

Determination of the optimal chirp-rate parameter $\alpha$ can be performed by a direct search in the assumed set of $\alpha$ values

$$
\hat{\alpha}_{\text {opt }}(m)=\arg \max _{\alpha \in \Lambda} H(m, \alpha ; \gamma)
$$

over the parameter space $\Lambda=\left[0, \alpha_{\max }\right]$ where $\alpha_{\max }$ is the chirp rate that corresponds to the TF plane diagonal $\alpha_{\max }=$ $2 \pi\left(1 / 2 T_{s}\right) /\left(N T_{s} / 2\right)=2 \pi /\left(N T_{s}^{2}\right)$, where $1 / 2 T_{s}$ is the maximal frequency that can be achieved with sampling rate $T_{s}$ within repetition time $T_{r}, T_{s}=T_{r} / N$. Direct search over a single parameter is nowadays considered as an acceptable computational burden. However, in the case when calculation time is critical, faster procedures should be used. For example, in the case of monocomponent signals embedded in a moderate noise, the LMS style algorithm can be employed. The optimal value of the chirp-rate parameter can be evaluated as

$$
\alpha_{i+1}(m)=\alpha_{i}(m)-\mu \frac{H\left(t, \alpha_{i}(m) ; \gamma\right)-H\left(t, \alpha_{i-1}(m) ; \gamma\right)}{\alpha_{i}(m)-\alpha_{i-1}(m)},
$$

where $\left[H\left(m, \alpha_{i}(m) ; \gamma\right)-H\left(m, \alpha_{i-1}(m) ; \gamma\right)\right] /\left[\alpha_{i}(m)-\alpha_{i-1}(m)\right]$ is used to estimate gradient of concentration measure and $\mu$ is the predefined step. This form of the algorithm has been implemented and applied for TF representations in [11]. A very fast (but sensitive to noise influence) technique for estimation of the chirp-rate parameters has been proposed in [13].

\subsubsection{Multicomponent signals}

Previously described procedure for determination of the adaptive chirp-rate parameter can be applied when reflected chirp can be represented as a monocomponent FM signal. Furthermore, the same procedure can be applied for multicomponent signals with the same or similar second derivatives of the signal phase since search for just one chirprate parameter should be performed. This situation corresponds to close scatterer points in the radar image with similar motion trajectories.

However, a modification is required in the case of several components, with different chirp rates. Namely, the previously described algorithm in this case would produce high concentration of dominant signal component, while the remaining components would be spread in the TF plane. The method proposed in [14] is based on calculation of an adaptive transform, as a weighted sum of the LPFTs,

$$
\begin{aligned}
& F_{A D}\left(\omega_{\tau}, m\right) \\
& \quad=\frac{1}{\int_{-\infty}^{\infty} H(m, \alpha ; \gamma) d \alpha} \int_{-\infty}^{\infty} F\left(\omega_{\tau}, m ; \alpha\right) H(m, \alpha ; \gamma) d \alpha,
\end{aligned}
$$

where weighted coefficients are proportional to the concentration measure. In our previous research this method had 
produced good results for signals with components of similar magnitudes. However, if signal components significantly differ in amplitude, the results are not satisfactory. Namely, signal components with smaller amplitude would be additionally attenuated. In order to avoid this drawback, we will use the following adaptive local polynomial FT:

$$
F_{A D}\left(\omega_{\tau}, m\right)=\sum_{i=1}^{P} F\left(\omega_{\tau}, m ; \alpha_{i}(m)\right),
$$

where the first adaptive frequency is estimated as

$$
\alpha_{1}(m)=\arg \max _{\alpha} H^{(0)}(m, \alpha ; \gamma)
$$

with $H^{(0)}(m, \alpha ; \gamma)=H(m, \alpha ; \gamma)$, given with (28) and set $i=1$. After detection of the first component's chirp rate, values of $H(m, \alpha ; \gamma)$ in a narrow zone around $\alpha_{1}(m)$ are neglected, and the search for the next maximum is performed. Each iteration in this procedure could be described into two steps:

$$
\begin{aligned}
H^{(i)}(m, \alpha ; \gamma) & = \begin{cases}H^{(i-1)}(m, \alpha ; \gamma) & \left|\alpha-\alpha_{i}(m)\right| \geq \Delta, \\
0 & \text { otherwise, }\end{cases} \\
\alpha_{i+1}(m) & =\arg \max _{\alpha} H^{(i)}(m, \alpha ; \gamma), \quad i=i+1 .
\end{aligned}
$$

This procedure should be stopped after the maximal value of $\arg \max _{\alpha} H^{(i)}(m, \alpha ; \gamma)$ becomes smaller than an assumed threshold. We set that the threshold is $25 \%$ of $\max _{\alpha} H^{(0)}(m, \alpha ; \gamma)$, that is, $25 \%$ of concentration measure before we start with peeling of components. Note that the parameter $\Delta$ should be selected carefully so that the next recognized component is not just a "side lobe" of the previous strong component. In the case when components have chirp rates close to each other, it is enough to recognize single chirp rate, since the proposed approach will improve concentration of all the components with similar chirp rates. In our experiments we assumed that the number of components with different chirp rates for considered radar chirp cannot be larger than 8 and we selected that $\Delta=\alpha_{\max } / 16=\pi /\left(8 N T_{s}^{2}\right)$. It produces accurate results in all of our experiments. Note that an alternative method for evaluation of the LPFT is proposed in [15].

\subsubsection{Combination of the results from various radar chirps}

In the case of radar signals we can assume that scatterers at close positions in the range/cross-range plane have similar motion parameters. It means that for chirps with similar chirp number we can take similar value of chirp-rate parameter. The chirp rate estimated for the $m$ th chirp can be used with a small error for the next chirp signal, without recalculating concentration measure. This simplified technique was accurate in simple simulated reflector geometry. In the case of complex reflector geometry, with numerous close components, inaccurate chirp-rate parameter estimates are obtained in several percents of chirps. Usage of one chirp rate for the next chirps causes the error propagation effect. Therefore, the concentration measure is calculated and chirp-rate parameter should be estimated for each chirp. In order to refine the results further, nonlinear filtering of the obtained chirp rates is performed. Assume that the chirp-rate parameter $\alpha(m)$ is estimated for each chirp. The nonlinear median filter can be calculated as

$$
\hat{\alpha}(m)=\operatorname{median}\{\alpha(m+i), i \in[-r, r]\}
$$

where $2 r+1$ is the width of the used median filter. Note that other filters with ability to remove impulse noise can be used here instead of the median filter like, for example, the $\alpha$-trimmed mean filters $[16,17]$.

\subsection{Second form: adaptive LPFT for regions of the radar image}

Methods for adaptive calculation of the radar image described so far propose evaluation of the adaptive parameter for each considered chirp and possible refinement by combining results obtained on close sensors. The implicit assumption was that the close points in the range/cross-range domain have similar chirp-rate parameters. In order to have more robust technique, that is able to deal with more challenging motion models, we propose alternative form of the adaptive LPFT with 2D optimization of chirp parameters. In defining this procedure, we keep in mind that relatively small portion of the radar image is related to the target. Consider just a part of the radar image above a threshold,

$$
I_{\varepsilon}\left(\omega_{\tau}, \omega_{m}\right)= \begin{cases}1 & \left|Q\left(\omega_{\tau}, \omega_{m}\right)\right|>\varepsilon \max \left\{\left|Q\left(\omega_{\tau}, \omega_{m}\right)\right|\right\} \\ 0 & \text { otherwise. }\end{cases}
$$

The region $I_{\mathcal{\varepsilon}}\left(\omega_{\tau}, \omega_{m}\right)$ can be separated into nonoverlapping regions

$$
I_{\mathcal{\varepsilon}}\left(\omega_{\tau}, \omega_{m}\right)=\bigcup_{i=1}^{p_{\varepsilon}} I_{i}\left(\omega_{\tau}, \omega_{m}\right)
$$

where $I_{i}\left(\omega_{\tau}, \omega_{m}\right) \cap I_{j}\left(\omega_{\tau}, \omega_{m}\right)=\varnothing$ for $i \neq j$. We assume that each region $I_{i}\left(\omega_{\tau}, \omega_{m}\right)$ is the largest one so that between any two points that belong to the same region $I_{i}\left(\omega_{\tau}, \omega_{m}\right)$ there exists a path that passes through points that belong to the region. Note that the number of separated regions $p_{\varepsilon}$ depends on selected threshold $\varepsilon$. By using the inverse 2D FT we can calculate signals associated with the region $I_{i}\left(\omega_{\tau}, \omega_{m}\right)$,

$$
q_{i}(m, \tau)=\operatorname{IFT}\left\{Q\left(\omega_{\tau}, \omega_{m}\right) I_{i}\left(\omega_{\tau}, \omega_{m}\right)\right\}, \quad i=1,2, \ldots, p_{\varepsilon} .
$$

Now, we can assume that signal $q_{i}(m, \tau)$ is generated by a single reflector. Then, we can perform optimization of each signal $q_{i}(m, \tau)$. Since this signal is already localized in the range/cross-range domain, we will not perform optimization for each $\tau$ or $m$, but only optimization with a single chirp 
function for each region $I_{i}\left(\omega_{\tau}, \omega_{m}\right)$,

$$
\begin{aligned}
& F_{i}\left(\omega_{\tau}, \omega_{m} ; \hat{\alpha}_{i}\right) \\
& \quad=\int_{-\infty}^{\infty} \sum_{m=0}^{M-1} q_{i}(m, \tau) \exp \left(-\frac{j \hat{\alpha}_{i} \tau^{2}}{2}-j \omega_{\tau} \tau-j \omega_{m} m\right) d \tau,
\end{aligned}
$$

where

$$
\hat{\alpha}_{i}=\arg \max _{\alpha} \frac{1}{\int_{-\infty}^{\infty} \sum_{m=0}^{M-1}\left|F_{i}\left(\omega_{\tau}, \omega_{m} ; \alpha\right)\right|^{\gamma} d \omega_{\tau}} .
$$

The radar image is calculated as a sum of the adaptive LPFT $F_{i}\left(\omega_{\tau}, \omega_{m} ; \hat{\alpha}_{i}\right):$

$$
F_{\varepsilon, A D}\left(\omega_{\tau}, \omega_{m}\right)=\sum_{i=1}^{p_{\varepsilon}} F_{i}\left(\omega_{\tau}, \omega_{m} ; \hat{\alpha}_{i}\right) .
$$

In our experiments we obtain very good results for $\varepsilon$ in a relatively wide range for numerous radar images.

However, additional optimization can be done based on the threshold $\varepsilon$. Here, a three-step technique for threshold selection is considered. In the first stage we consider various thresholds $\varepsilon \in \Xi$ and calculate $F_{\varepsilon, A D}\left(\omega_{\tau}, \omega_{m}\right)$ for each threshold from the set. Then, we calculate the optimal LPFT as $F_{\varepsilon, A D}\left(\omega_{\tau}, \omega_{m}\right)$ that achieves the best concentration over $\varepsilon \in \Xi$. Since, by introducing the threshold value, we remove a part of the range/cross-range plane (see (36)) the energy of $F_{\varepsilon, A D}\left(\omega_{\tau}, \omega_{m}\right)$ should be normalized to the energy of signal above the specific threshold,

$$
\begin{gathered}
F_{\varepsilon, A D}^{\prime}\left(\omega_{\tau}, \omega_{m}\right)=\frac{F_{\varepsilon, A D}\left(\omega_{\tau}, \omega_{m}\right)}{\sqrt{\int_{-\infty}^{\infty} \sum_{m=0}^{M-1}\left|Q\left(\omega_{\tau}, \omega_{m}\right)\right|^{2} I_{\mathcal{\varepsilon}}\left(\omega_{\omega}, \omega_{m}\right) d \omega_{t}}}, \\
\hat{\varepsilon}=\arg \max _{\varepsilon \in \Xi} \frac{1}{\int_{-\infty}^{\infty} \sum_{m=0}^{M-1}\left|F_{\varepsilon, A D}^{\prime}\left(\omega_{\tau}, \omega_{m}\right)\right|^{\gamma} d \omega_{t}} .
\end{gathered}
$$

In this procedure the transforms, $F_{\varepsilon, A D}\left(\omega_{\tau}, \omega_{m}\right), \varepsilon \in \Xi$, are compared under unequal conditions since they are obtained with various thresholds $\varepsilon$ and they could have different number of recognized components. Obtained adaptive transform $F_{\varepsilon, A D}\left(\omega_{\tau}, \omega_{m}\right)$ could be worse concentrated than a particular $F_{\varepsilon, A D}^{\prime}\left(\omega_{\tau}, \omega_{m}\right)$ from the considered set of $\varepsilon$ values. However, this radar image is close to the best one and a small additional manual adaptation around the estimated $\hat{\varepsilon}$ could be performed in the third stage of this procedure. In our experiments we obtain that $\hat{\varepsilon}$ is underestimated. Thus, additional search could be performed over higher values of $\varepsilon$.

\section{NUMERICAL EXAMPLES}

Several numerical examples will be presented here to justify the presented approach. Examples 1-4 are generic signals representing one received radar chirp that proves that the adaptive LPFT can be used to produce highly concentrated TF representation for following 1D signals: linear FM, sinusoidal FM, multicomponent signal with similar chirp rates, and multicomponent signal with different chirp rates. Examples 5 and 6 demonstrate that the adaptive LPFT optimized for each chirp signal with filtering data produced by adjacent radar chirps gives accurate results. Example 7 illustrates the second adaptive LPFT algorithm with optimization for detected regions of interest in radar image.

Example 1. The first signal that will be considered is a linear FM signal $f(t)=\exp \left(j 64 \pi t^{2} / 2\right)$ embedded in Gaussian noise with variance $\sigma^{2}=1$. The signal is sampled with $\Delta t=$ $1 / 128$ second. The FT of the windowed signal with a Hanning window of the width $T=2$ second is shown in Figure 2(a). It can be seen that the FT is spread. Thus, if this signal is a part of the received signals reflected from a target, we will obtain a defocused radar image. Results obtained with narrower Hanning windows are given in Figure 2(b). Improvement could be observed from this figure, but generally speaking it is slight. The concentration measure (28) for $\gamma=1$ is presented in Figure 2(c), with marked detected chirp-rate parameter. Finally, adaptive LPFT is given in Figure 2(d) calculated for parameter $\alpha$ for which the concentration measure given in Figure 2(c) is maximized. Significant improvement achieved by the LPFT is obvious.

Example 2. The second signal is a more complex sinusoidal FM signal: $f(t)=\exp (j 16 \sin (2 \pi t))$. Signal sampling and noise environment are the same as in Example 1. The FTs with wide and narrow windows around a given time instant (STFT), [18], are depicted in Figures 3(a) and 3(b). This STFT illustration for fixed instant corresponds to the radar image for considered $m$. It can be used to estimate radar image depending on different chirp rates. Again we can see that for each instant this representation is spread in frequency domain. It means that the radar image obtained based on the FT for signal of this form will be defocused. Adaptive LPFT with a single chirp rate, calculated for each instant, is given in Figure 3(c). A significant improvement is achieved. Also, it can be noticed that the representation is not ideal in the region with higher order derivatives. These derivatives can be removed by employing higher order LPFT form [7-9]. Adaptive chirp rate is given in Figure 3(d).

Example 3. A three-component signal: $f(t)=\exp \left(j 22 \pi t^{2}+\right.$ $j 48 \pi t)+\exp \left(j 32 \pi t^{2}\right)+\exp \left(j 42 \pi t^{2}-j 48 \pi t\right)$ is considered next. The STFT with a wide and a narrow window is given in Figures 4(a) and 4(b). The adaptive LPFT calculated as in the case of monocomponent signal is given in Figure 4(c). It can be seen that the concentration is improved for all three components. Component in the middle is enhanced the best, but other components with similar chirp rates are also improved. The adaptive parameter is given in Figure 4(d). This case corresponds to a signal obtained from several scatterers in the same cross-range with similar chirp rates. Difference in chirp rates of these components in fact is not so small, it is $30 \%$ of the chirp rate of middle component. It is a realistic case for numerous targets in practice. We can see that concentration of all components is satisfactory. It can also be seen that accuracy of this procedure is not affected by the distance between scatterers points. The same accuracy is achieved for the left part of Figure 4(c), where we assume that scatterers 


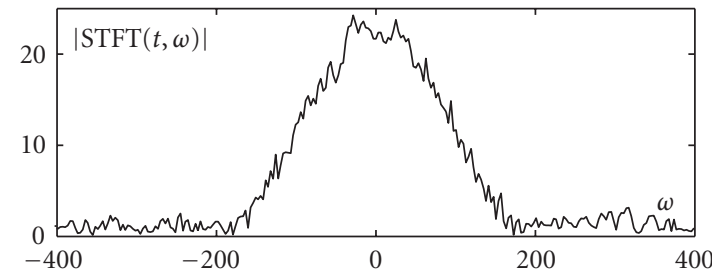

(a)

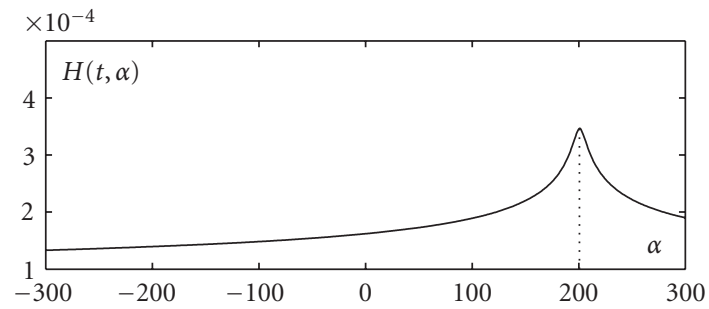

(c)

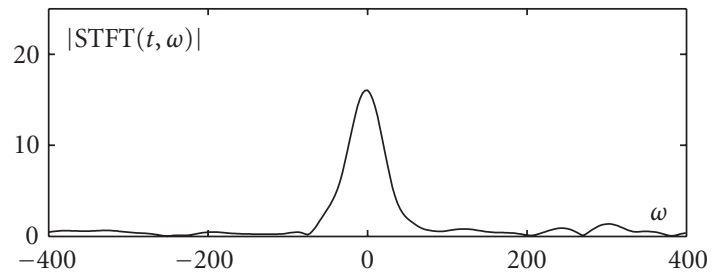

(b)

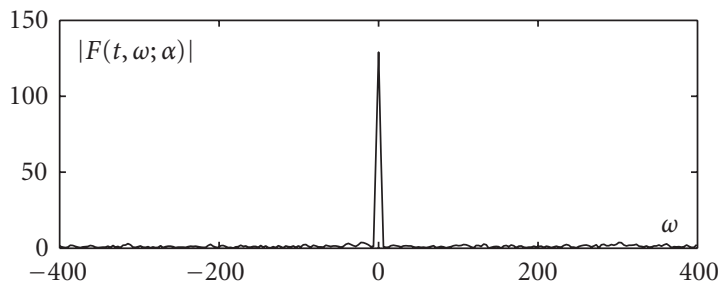

(d)

FIGURE 2: Spectral analysis of the linear FM signal: (a) FT with a wide window; (b) FT with a narrow window; (c) concentration measure; (d) adaptive LPFT.

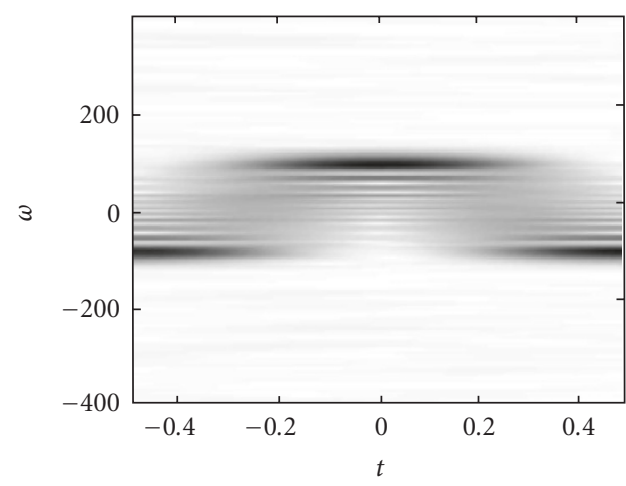

(a)

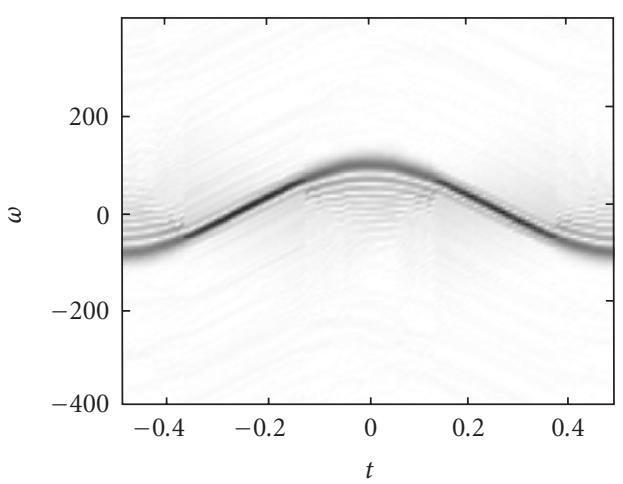

(c)

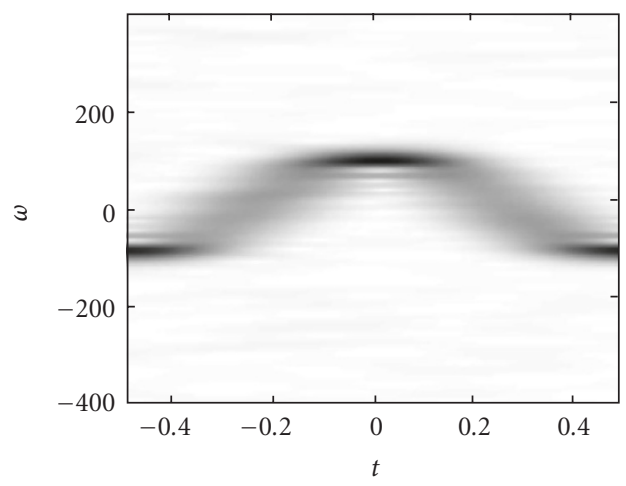

(b)

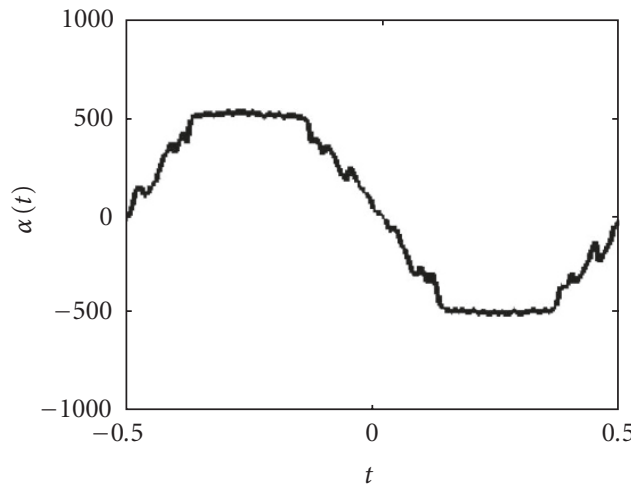

(d)

FIGURE 3: Time-frequency analysis of the sinusoidal FM signal: (a) STFT with a wide window; (b) STFT with a narrow window; (c) adaptive LPFT; (d) adaptive chirp-rate parameter. 


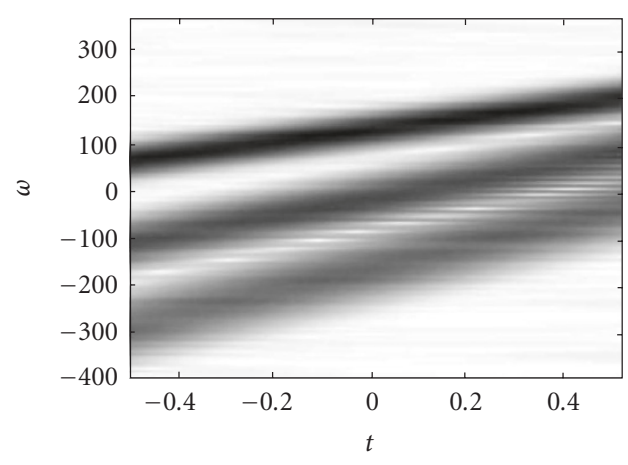

(a)

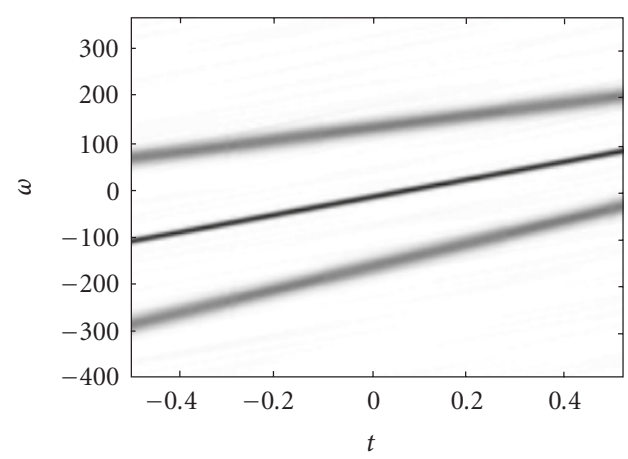

(c)

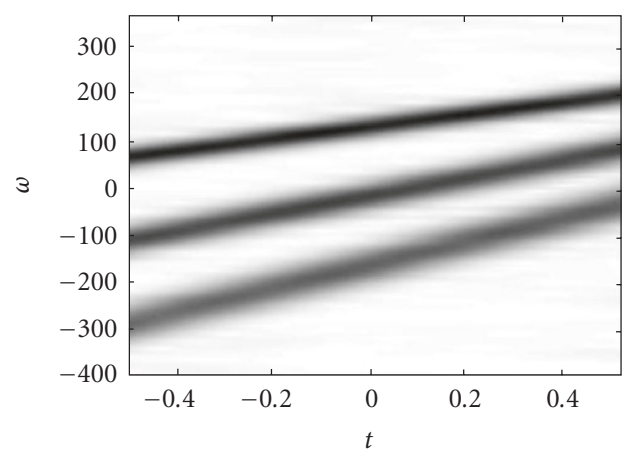

(b)

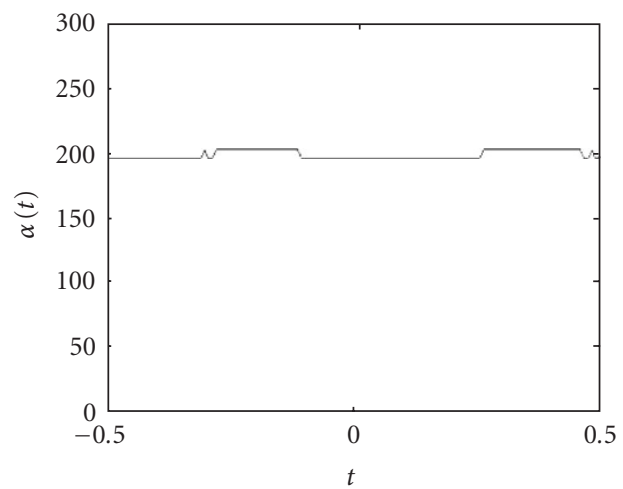

(d)

FIGURE 4: Time-frequency analysis of multicomponent signal: (a) STFT with a wide window; (b) STFT with a narrow window; (c) adaptive LPFT; (d) adaptive chirp-rate parameter.

are far from each other, as well as in the right part of this illustration, where it can be assumed that scatterers are close to each other.

Example 4. A three-component signal: $f(t)=\exp \left(j 11 \pi t^{2}+\right.$ $j 48 \pi t)+\exp \left(j 32 \pi t^{2}\right)+\exp \left(j 67 \pi t^{2}-j 48 \pi t\right)$ is considered. However, in this case the chirp rates of components are quite different (difference between chirp rates is more than $60 \%$ of chirp rate of middle component). The STFT is given in Figure 5(a), while the "adaptive" transform, assuming that signal has single chirp rate, is given in Figure 5(b). It can be seen that in each instant, the transform is adjusted to one component, while other components remain spread. For $t<0.3$, the LPFT is highly concentrated for middle component, but when components are close to each other (it corresponds to close scatterers) the adaptive chirp rate several times switches between components. The adaptive weighted LPFT (32) is given in Figure 5(c). It can be seen that all components have improved concentration and that concentration is not influenced by distance between scatterers. Detected adaptive chirp rates are given in Figure 5(d).

Example 5. Simulated radar target setup according to the experiment in [4] is considered. The reflectors are at the positions $(x, y)=\{(-2.5,1.44),(0,1.44),(2.5,1.44),(1.25$, $-0.72),(0,2.88),(-1.25,0.72)\}$ in meters. High resolution radar operates at the frequency $f_{0}=10.1 \mathrm{GHz}$, with a bandwidth of linear FM chirps $B=300 \mathrm{MHz}$ and pulse chirp repetition time $T_{r}=15.6 \mathrm{~ms}$. The target is at $2 \mathrm{~km}$ distance from the radar, and rotates at $\omega_{R}=4^{\circ} / \mathrm{s}$. The nonlinear rotation with frequency $\Omega=0.5 \mathrm{~Hz}$ and amplitude $A=1.25^{\circ} / \mathrm{s}$ is superimposed, $\omega_{R}(t)=\omega_{R}+A \sin (2 \pi \Omega t)$. The FT-based image of radar target is depicted in Figure 6(a). The radar image obtained by using the adaptive LPFT calculated for each chirp separately is presented in Figure 6(c), while the adaptive parameter for each chirp signal is given in Figure 6(b). It can be seen that the adaptive parameter linearly varies between the limits of the target. However, the impulse like errors in estimation of the chirp rate can be observed from Figure 6(b). It suggests that improvement of the results can be achieved by filtering chirp-rate parameters.

Example 6. In this example we consider a B727 radar data. The FT-based image is presented in Figure 7(a). It can be seen that the radar image is defocused, thus causing the problem to extract the target. However, radar imaging based on the adaptive LPFT determined for each radar chirp produces a significant improvement in the signal representation, 


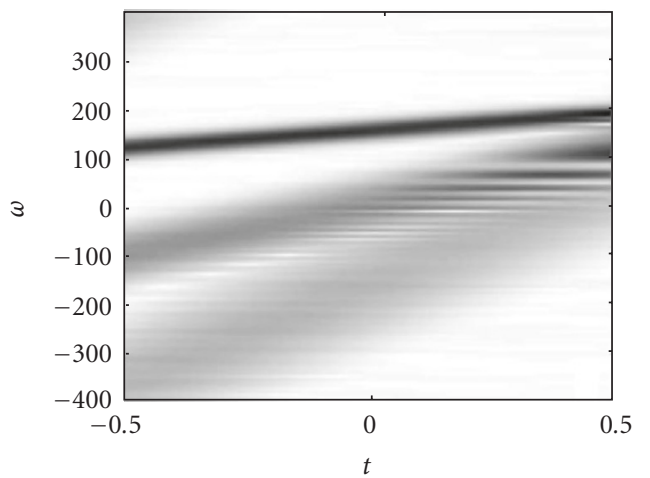

(a)

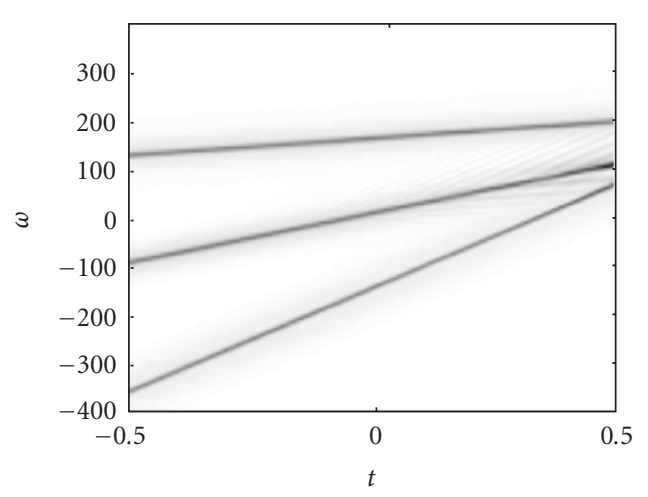

(c)

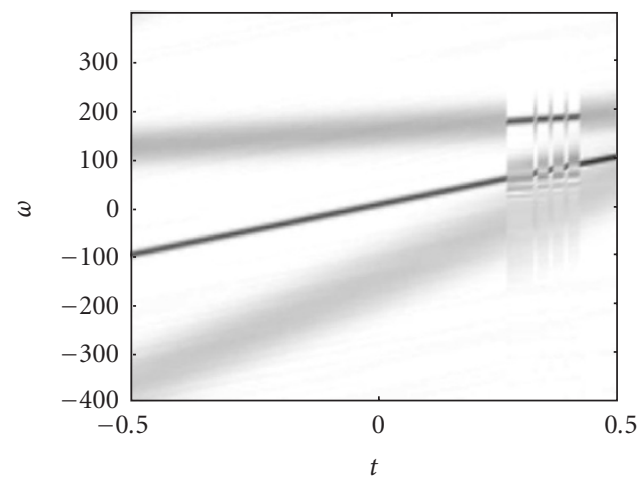

(b)

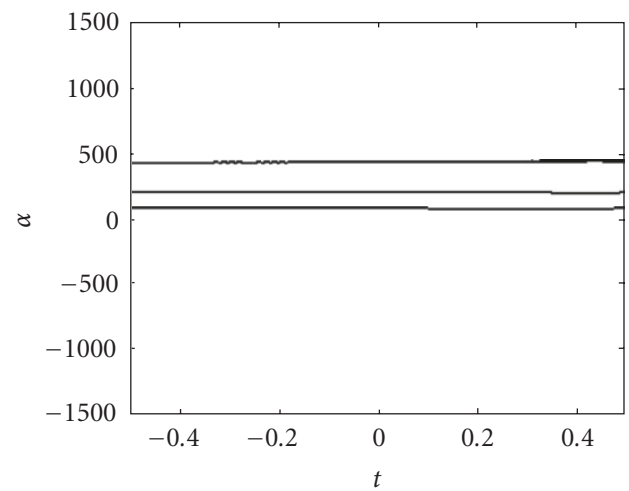

(d)

FIGURE 5: Time-frequency analysis of multicomponent signal: (a) STFT with a wide window; (b) LPFT with a single chirp-rate parameter estimated in each instant; (c) weighted adaptive LPFT; (d) estimated chirp rates.

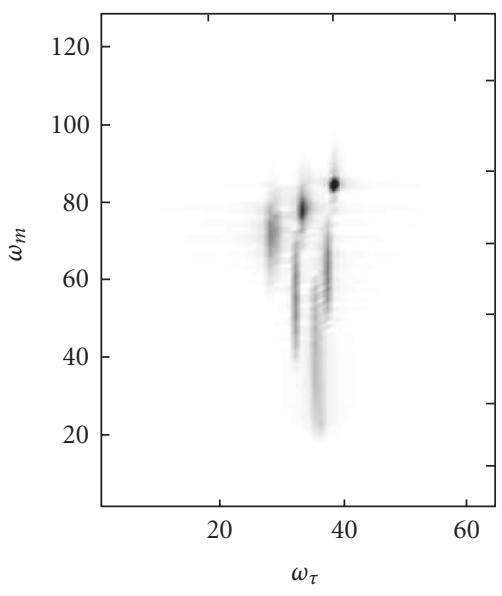

(a)

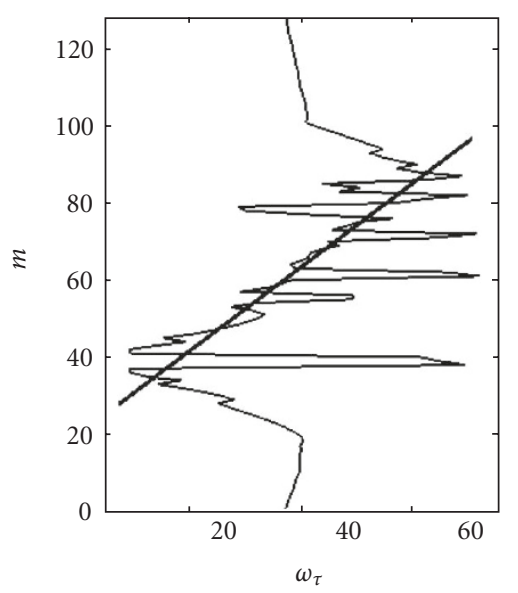

(b)

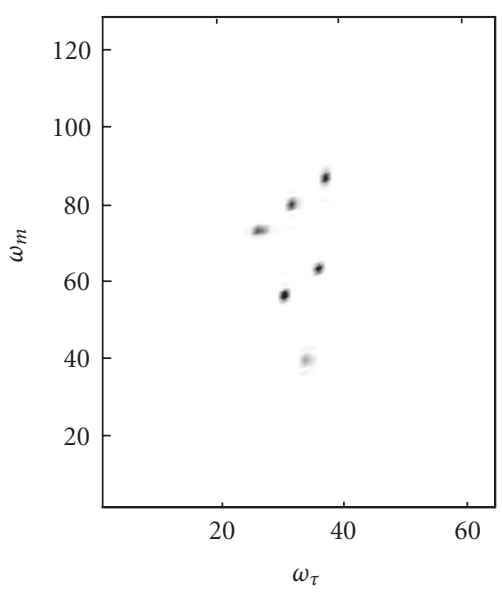

(c)

FIgURE 6: Simulated radar image: (a) results obtained by the FT; (b) adaptive chirp-rate parameter as function of $m$ (thick line is linear approximation); (c) radar image based on the adaptive LPFT. 


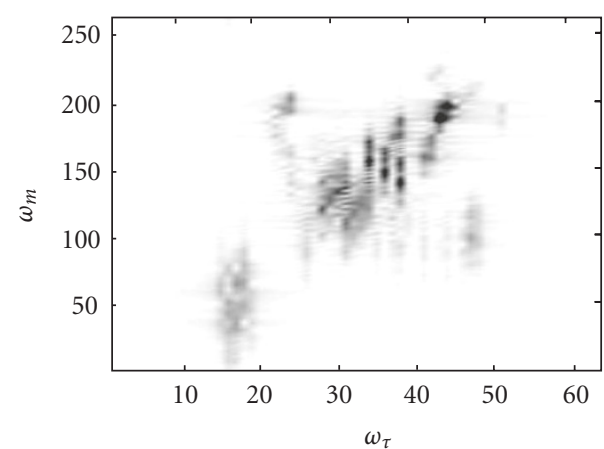

(a)

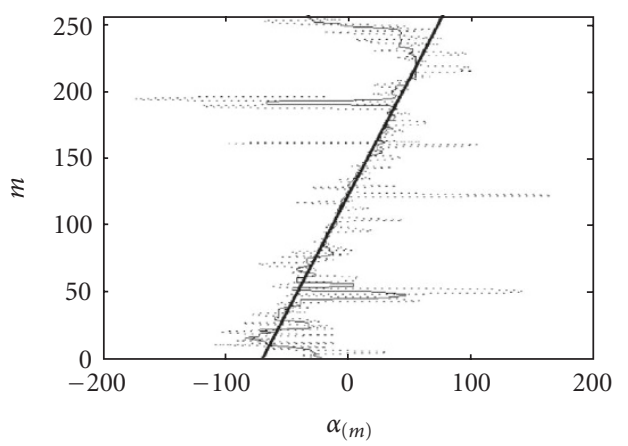

(c)

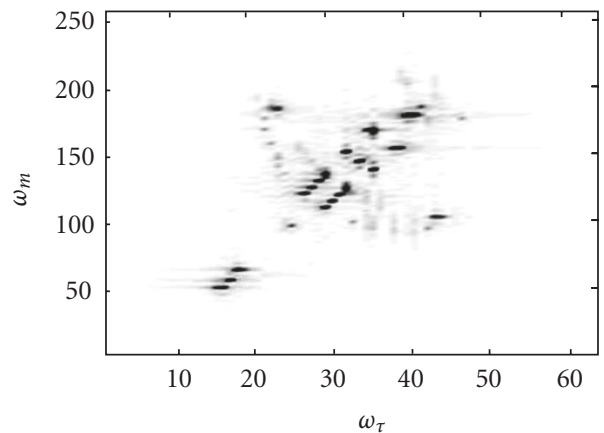

(b)

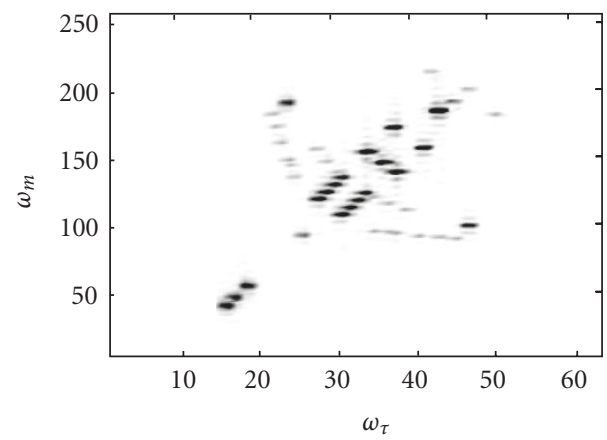

(d)

FIGURE 7: B727 radar image: (a) results obtained by the FT-based method; (b) adaptive LPFT method; (c) adaptive chirp rate (dotted line), filtered adaptive chirp rate (light solid line), linear interpolation of filtered data (bold solid line); (d) adaptive LPFT with interpolated data.

Figure 7(b). In order to obtain better results for close reflectors, we consider the adaptive chirp-rate parameter depicted in Figure 7(c) as a dotted line. We expected that removing impulse-like disturbances will produce better results. To this aim median filtering of the adaptive parameter is performed. In addition, the linear interpolation of estimated chirp rates is performed (linear interpolation is depicted with thick line in Figure 7(c)). The result obtained with these parameters is depicted in Figure 7(d). It is better than its counterpart in Figure 7(b) except for nose reflectors. A possible reason is in fact that the received signal corresponding to these scatterers can have higher order polynomial in the signal phase. The higher order LPFT forms [7-9] could be used for these scatterers points (see Section 3.1.2).

Example 7. In this example we consider the same target as in Example 5. The main difference in this example is in complex motion pattern that cannot be modeled with just a rotation. The radar image calculated by using the $2 \mathrm{D}$ FT is presented in Figure 8(a). Region of interest $I_{\varepsilon}\left(\omega_{\tau}, \omega_{m}\right)$ is determined by (36) with the threshold set to $\varepsilon=0.05$. Three separated regions are detected in radar image denoted in Figure 8(b) in different shades of gray. The region denoted with 1 corresponds to three radar scatterers. Since these three scatterers move in a similar manner, concentration of these com- ponents is significantly improved (see Figure 8(c)) with respect to the radar image calculated with 2D FT. Region denoted with number 3 corresponds to two radar scatterers. In this case concentration of one of components from the region is improved, while other component remains spread. The reason is in fact that these close scatterers move in a quite different manner. When we apply threshold $\varepsilon=0.2$, we obtain 6 regions of interest that correspond to 6 radar scatterers (Figure $8(\mathrm{~d})$ ). The resulting radar image is focused for all scatterers (Figure 8(e)). The threshold $\varepsilon$ could be set in an empirical manner. However, a procedure for threshold optimization could be very helpful. Concentration measure of adaptive LPFT for various threshold levels is depicted in Figure $9(\mathrm{a})$ and obtained value in the optimization procedure is $\hat{\varepsilon} \approx 0.155$. The LPFT form with adaptive threshold is shown in Figure 9(b). It can be seen that radar image obtained in Figure 9(a) is slightly worse than radar image with additionally adjusted threshold (Figure 8(e)).

\section{CONCLUSION}

The adaptive local polynomial Fourier transform-based method for enhancement of defocused radar images has been proposed. Adaptive parameters in the transform are obtained 


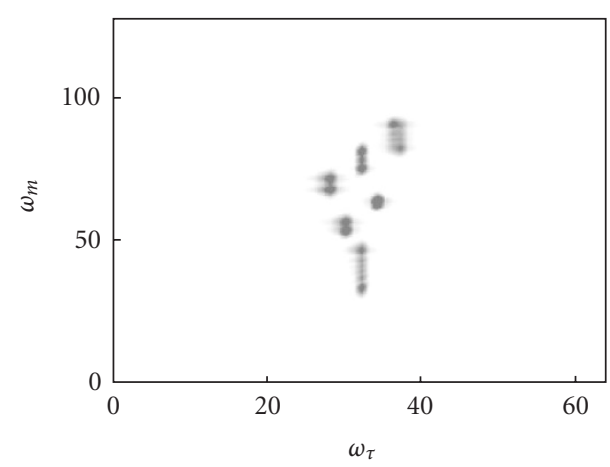

(a)

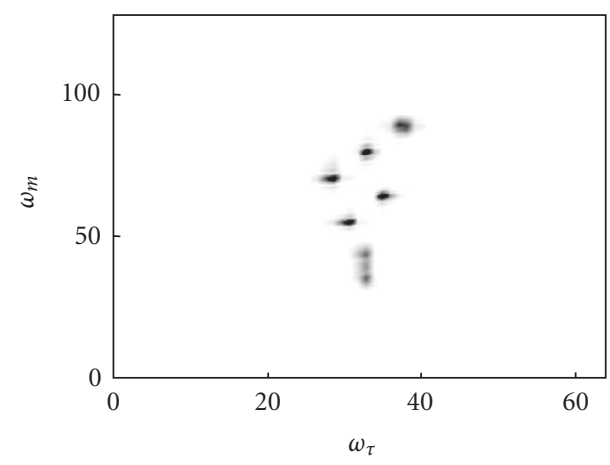

(c)

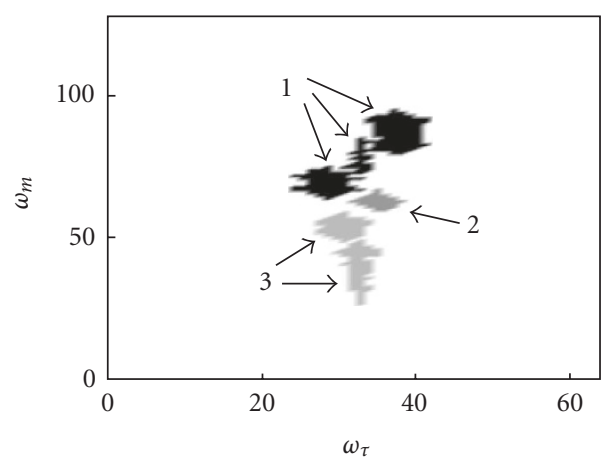

(b)

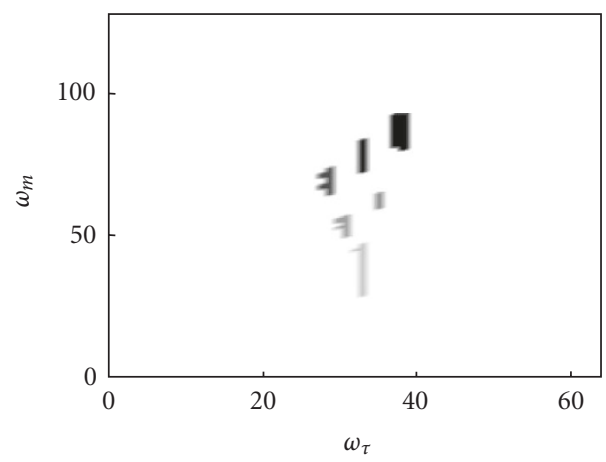

(d)

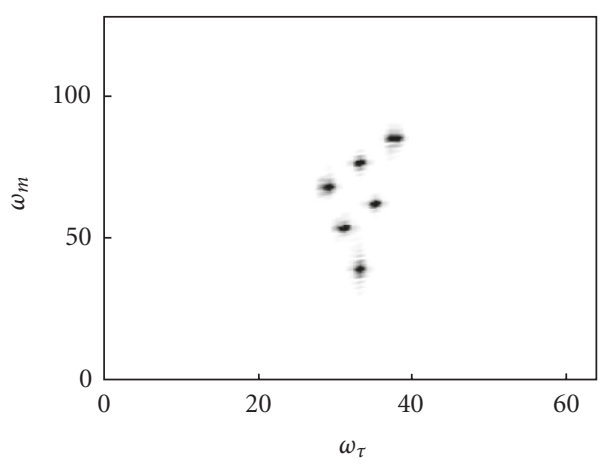

(e)

FIGURE 8: Simulated radar image with complicated motion pattern: (a) results obtained by the FT; (b) regions of interest $I_{\varepsilon=0.05}\left(\omega_{t}, \omega_{m}\right)$ with three recognized separated regions; (c) adaptive LPFT based on region optimization with $\varepsilon=0.05, F_{\varepsilon=0.05}\left(\omega_{t}, \omega_{m}\right)$; (d) regions of interest $I_{\varepsilon=0.20}\left(\omega_{t}, \omega_{m}\right)$ with six recognized separated regions; (e) adaptive LPFT based on region optimization with $\varepsilon=0.20, F_{\varepsilon=0.20}\left(\omega_{t}, \omega_{m}\right)$.

by using a simple concentration measure. For monocomponent and multicomponent signals with similar chirp rates, a single chirp-rate parameter is estimated for each chirp. For multicomponent signals with different chirp rates, an adaptive weighted local polynomial FT should be employed. It has been shown that the ISAR images could be improved by combining results achieved from various chirps. For targets with very complex motion pattern, separation of the radar image in regions of interests and optimization of the radar signal within regions are proposed. 


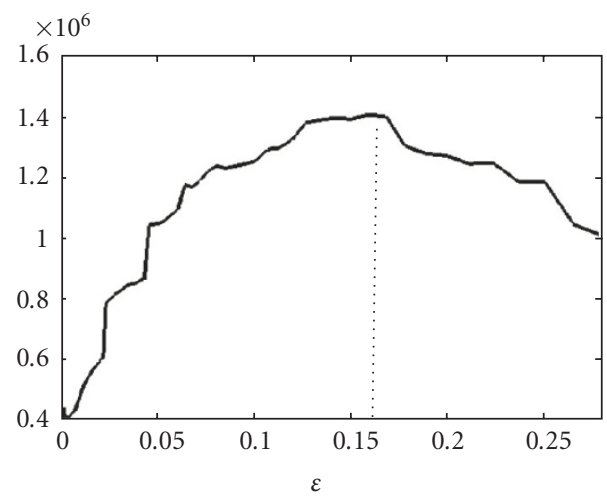

(a)

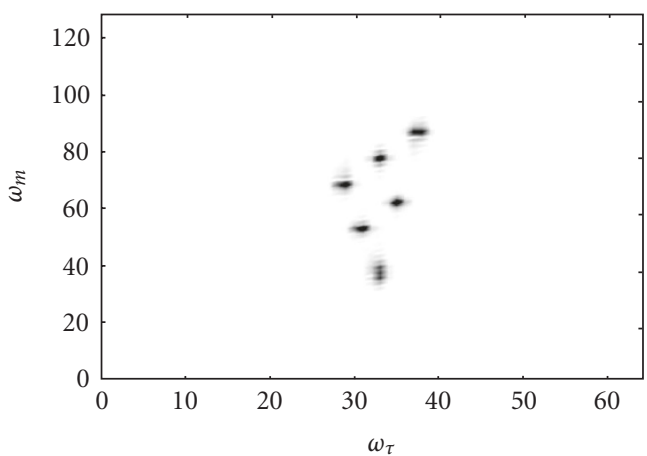

(b)

FIGURE 9: Adaptive LPFT with adaptive threshold: (a) concentration measure for various threshold levels; optimal threshold value is depicted with dotted line; (b) adaptive LPFT with adaptive threshold.

\section{REFERENCES}

[1] Y. Wang, H. Ling, and V. C. Chen, "ISAR motion compensation via adaptive joint time-frequency technique," IEEE Transactions on Aerospace and Electronic Systems, vol. 34, no. 2, pp. 670-677, 1998.

[2] S. Barbarossa, A. Scaglione, and G. B. Giannakis, "Product high-order ambiguity function for multicomponent polynomial-phase signal modeling," IEEE Transactions on Signal Processing, vol. 46, no. 3, pp. 691-708, 1998.

[3] A. Quinquis, C. Ioana, and E. Radoi, "Polynomial phase signal modeling using warping-based order reduction," in Proceedings of IEEE International Conference on Acoustics, Speech, and Signal Processing (ICASSP '04), vol. 2, pp. 741-744, Montreal, Quebec, Canada, May 2004.

[4] S. K. Wong, E. Riseborough, and G. Duff, "Experimental investigations on the distortion of ISAR images using different radar waveforms," Tech. Mem. DRDC Ottawa TM 2003-1996, Defence Research \& Development Canada, Ottawa, Ontario, Canada, 2003.

[5] S. K. Wong, G. Duff, and E. Riseborough, "Distortion in the ISAR (inverse synthetic aperture radar) images from moving targets," in Proceedings of IEEE International Conference on Image Processing (ICIP'04), vol. 1, pp. 25-28, Singapore, October 2004.

[6] T. Thayaparan, G. Lampropouols, S. K. Wong, and E. Riseborough, "Application of adaptive joint time-frequency algorithm for focusing distorted ISAR images from simulated and measured radar data," IEE Proceedings - Radar, Sonar and Navigation, vol. 150, no. 4, pp. 213-220, 2003.

[7] V. Katkovnik, "A new form of the Fourier transform for timevarying frequency estimation," Signal Processing, vol. 47, no. 2, pp. 187-200, 1995.

[8] V. Katkovnik, "Local polynomial periodogram for timevarying frequency estimation," South African Statistical Journal, vol. 29, no. 2, pp. 169-198, 1995.

[9] LJ. Stanković and S. Djukanović, "Order adaptive local polynimial FT based interference rejection in spread spectrum communication systems," in Proceedings of IEEE International Symposium on Intelligent Signal Processing (WISP '03), Budapest, Hungary, September 2003.

[10] R. G. Baraniuk, P. Flandrin, A. J. E. M. Jensen, and O. J. J. Michel, "Measuring time-frequency information content using Rényi entropy," IEEE Transactions on Information Theory, vol. 47, no. 4, pp. 1391-1409, 2001.

[11] LJ. Stanković, "A measure of some time-frequency distributions concentration," Signal Processing, vol. 81, no. 3, pp. 621631, 2001.

[12] T.-H. Sang and W. J. Williams, "Rényi information and signaldependent optimal kernel design," in Proceedings of IEEE International Conference on Acoustics, Speech, and Signal Processing (ICASSP '95), vol. 2, pp. 997-1000, Detroit, Mich, USA, May 1995.

[13] I. Djurović and LJ. Stanković, "Moments of multidimensional polynomial FT," IEEE Signal Processing Letters, vol. 11, no. 11, pp. 879-882, 2004.

[14] M. Daković, I. Djurović, and LJ. Stanković, "Adaptive local Fourier transform," in Proceedings of the 11th European Signal Processing Conference (EUSIPCO '02), vol. 2, pp. 603-606, Toulouse, France, September 2002.

[15] Y. Wei and G. Bi, "Efficient analysis of time-varying multicomponent signals with modified LPTFT," EURASIP Journal on Applied Signal Processing, vol. 2005, no. 8, pp. 1261-1268, 2005.

[16] I. Pitas and A. N. Venetsanopoulos, Nonlinear Digital Filters: Principles and Applications, Kluwer Academic, Boston, Mass, USA, 1990.

[17] I. Djurović, LJ. Stanković, and J. F. Böhme, "Robust Lestimation based forms of signal transforms and timefrequency representations," IEEE Transactions on Signal Processing, vol. 51, no. 7, pp. 1753-1761, 2003.

[18] J. B. Allen and L. R. Rabiner, "A unified approach to shorttime Fourier analysis and synthesis," Proceedings of the IEEE, vol. 65, no. 11, pp. 1558-1564, 1977. 
Igor Djurović was born in Montenegro in 1971. He received the B.S., M.S., and Ph.D. degrees in electrical engineering from the University of Montenegro, in 1994, 1996, and 2000, respectively. During 2002 he was on leave at the Department of Mechanical and System Engineering, Kyoto Institute of Technology, Japan, as the Japan Society for the Promotion of Science Fellow. He spent one month in the AIIA Laboratory, Aristo-

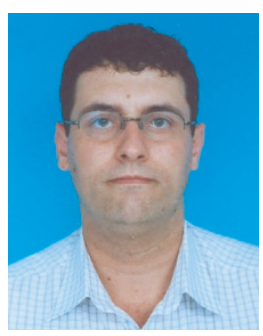
tle University, Thessaloniki, during 1999. Also, he spent one month in the Signal Theory Group, Ruhr University, Bochum, Germany, in 2002, and visited and joined research with ENSIETA, Brest, France, during 2003. He was awarded with biannual award of the National Academy of Science and Art of Montenegro for the Young Scientist with the best achievements in the technical sciences in Montenegro (2000-2002). He published over 70 papers in international scientific journals and conferences. He contributed to Time-Frequency Signal Analysis and Processing, Elsevier Science, 2003.

Thayananthan Thayaparan earned a B.S. degree with honors in physics from the University of Jaffna, Srilanka, in 1987, an M.S. degree in physics from the University of Oslo, Norway, in 1991, and a Ph.D. degree in atmospheric physics from the University of Western Ontario, Canada, in 1996. From 1996 to 1997, he was employed as a Postdoctoral Fellow at the University of Western Ontario. In 1997, he joined the Defence

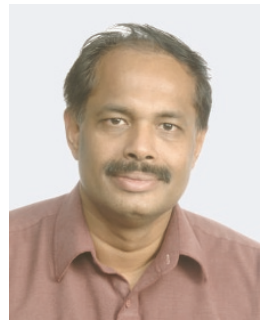
Research Establishment Ottawa (DREO), Department of National Defence, Canada, as a Defence Scientist. His research interests include, inter alia, time-frequency analysis, applications to radar signal and image processing, inverse synthetic aperture radar (ISAR), synthetic aperture radar (SAR), noncooperative target recognition (NCTR), moving target detection (MTD), ATR, meteors and ionosphere clutter in HF radar, and winds and waves in the middle atmosphere using MF and VHF meteo radars.

Ljubiša Stanković was born in Montenegro in 1960 . He received the B.S. degree in EE from the University of Montenegro, Podgorica, in 1982, the M.S. degree in EE in 1984 from the University of Belgrade. As a Fulbright Grantee, he spent 1984/1985 academic year at theWorcester Polytechnic Institute, Worcester, Mass. Since 1982, he has been on the faculty at the University of Montenegro, where he holds position of

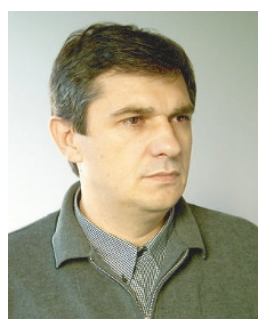
a Full Professor since 1995. During 1997/1998 and 1999, he was on leave at the Ruhr-University Bochum, Bochum, Germany, supported by the Alexander von Humboldt Foundation. At the beginning of 2001, he spent a period of time at the Technische Universiteit Eindhoven, Eindhoven, the Netherlands, as a Visiting Professor. He published about 270 technical papers, more than 80 of them in leading international journals, mainly the IEEE editions. He received the Highest State Award of the Republic of Montenegro in 1997 for scientific achievements. He is a Member the IEEE Signal Processing Society's Technical Committee on Theory and Methods, a Member of the Yugoslav Engineering Academy, and a Member of the National Academy of Science and Art of Montenegro (CANU). He is the Rector of the University of Montenegro since 2003. 